\title{
A microscopic endoscope
}

Microscopic optical devices make their way into living cells.

More than four years ago we highlighted work from two groups on microscopic optical tools and pronounced that resulting devices "... will change the way some scientists conduct experiments” (Evanko, 2007).

Although such tools have yet to make their way into the hands of biologists, we are seeing progress in the development of tools that could begin to have practical value to biologists. Peidong Yang from the University of California at Berkeley, one of the authors we previously highlighted, now reports how his group converted a light-carrying nanowire into a microscopic endoscope for single living cells (Yan et al., 2011). But as suggested by the time elapsed from the original report, this development was not trivial.

Yang recalls, "When we demonstrated nanowire wave-guiding and nonlinear mixing in solution, I was very excited that now we have these nanoscopic light sources in solution, and I thought, 'Let's go and test it in living cells.' Unfortunately this turned out to be harder than expected.

The first problem they ran into was the low mechanical stability of the unsupported nanowires. In their prior Nature paper they used optical tweezers to hold potassium niobate nanowires and deliver infrared light into them. The nonlinear mixing capability of the potassium niobate converted the infrared light into visible light that emanated from the tip. But the optical trapped nanowires were not sturdy enough to poke the probes into cells. Instead, Yang reasoned they had to physically mount the nanowires onto something that could exert sufficient force. They tried many alternatives but settled on coupling a tin dioxide nanowire that functions as a simple subwavelength waveguide to the end of an etched optical fiber.

The optical fiber provided good mechanical stability, but unlike with the optical trap, light transmission between the fiber and nanowire required careful refractive index matching to avoid large optical losses at the junction. Yang recalls, "To get these nanowires into a robust stable platform took us awhile, but then progress was almost spontaneous." They showed that unlike insertion of a tapered optical-fiber tip into cells, insertion of the 100-nanometer-diameter nanowire did not induce cell death or other

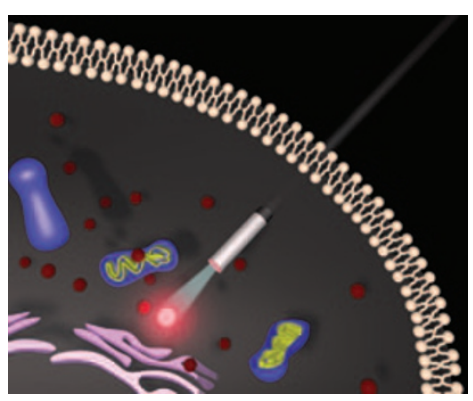

Illustration of a nanowire-based endoscope penetrating a living cell and illuminating a single quantum dot. Image courtesy of P. Yang.

obvious cellular stress over the course of the experiment.

The resulting nanowire probes have two major capabilities: localized imaging and localized delivery. To demonstrate localized delivery the researchers attached quantum dots to the nanowire tip using photocleavable linkers cleavable by external UV light illumination of the nanowire inside the cell. "We could place quantum dots where we wanted them in the cytoplasm or the nucleus," says Yang.

After delivery of the quantum dots they could use the nanowire probe to selectively illuminate single dots or to collect emitted light from one quantum dot cluster. In both scenarios the region of the cell being probed is a tiny volume. This has the benefit of reducing unwanted signals from cellular autofluorescence or other sources distant from the tip. Functionalization of the probe tip itself with a fluorescent sensor can additionally confine the signal and ensure measurement of only local changes. In a preliminary experiment the authors demonstrated this in microdroplets.

Yang is hopeful that additional applications of these nanowire probes can be developed and that biologists will find them useful. One hope is that the highly precise point detection these probes allow can be used for three-dimensional multiplex imaging of signals inside living cells. But Yang says, "It isn't going to be trivial."

\section{Daniel Evanko}

\section{RESEARCH PAPERS}

Evanko, D. Incredible shrinking optics. Nat. Methods 4, 683 (2007).

Yan, R. et al. Nanowire-based single-cell endoscopy. Nat. Nanotechnol. advance online publication (18 December 2011). 